Xuemei Zhang* and Meiqiang Feng

\title{
Boundary blow-up solutions to the Monge-Ampère equation: Sharp conditions and asymptotic behavior
}

https://doi.org/10.1515/anona-2020-0023

Received December 12, 2018; accepted March 4, 2019.

Abstract: Consider the boundary blow-up Monge-Ampère problem

$$
M[u]=K(x) f(u) \text { for } x \in \Omega, u(x) \rightarrow+\infty \text { as } \operatorname{dist}(x, \partial \Omega) \rightarrow 0 .
$$

Here $M[u]=\operatorname{det}\left(u_{x_{i} x_{j}}\right)$ is the Monge-Ampère operator, and $\Omega$ is a smooth, bounded, strictly convex domain in $\mathbb{R}^{N}(N \geq 2)$. Under $K(x)$ satisfying appropriate conditions, we first prove that the boundary blow-up MongeAmpère problem has a strictly convex solution if and only if $f$ satisfies Keller-Osserman type condition. Then the asymptotic behavior of strictly convex solutions to the boundary blow-up Monge-Ampère problem is considered under weaker condition with respect to previous references. Finally, if $f$ does not satisfy KellerOsserman type condition, we show the existence of strictly convex solutions under different conditions on $K(x)$. The proof combines standard techniques based upon the sub-supersolution method with non-standard arguments, such as the Karamata regular variation theory.

Keywords: Monge-Ampère equation; boundary blow up; sub-supersolution method; sharp conditions; strictly convex solution; asymptotic behavior

MSC: $35 \mathrm{~J} 60,35 \mathrm{~J} 96$

\section{Introduction}

Monge-Ampère problems are fully nonlinear problems, which can describe Weingarten curvature, or reflector shape design (see [1]). In the past years, increasing attention has been paid to the study of Monge-Ampère problems by various approaches. We list here, for example, papers [2-14]. The other recent results concerning fully nonlinear uniformly elliptic equations can be found in [15-20]

In this article, we consider the boundary blow-up problem for the Monge-Ampère equation

$$
M[u]=K(x) f(u) \text { in } \Omega, u=+\infty \text { on } \partial \Omega,
$$

where $M[u]=\operatorname{det}\left(u_{x_{i} x_{j}}\right)$ is the Monge-Ampère operator, $\Omega$ is a smooth, bounded, strictly convex domain in $\mathbb{R}^{N}(N \geq 2)$, and $K(x), f(u)$ are smooth positive functions. The boundary blow-up condition $u=+\infty$ on $\partial \Omega$ means

$$
u(x) \rightarrow+\infty \text { as } \operatorname{dist}(x, \partial \Omega) \rightarrow 0 .
$$

We aim to study the existence and asymptotic behavior of strictly convex solution to (1.1). Suppose $K(x)$ and $f(u)$ satisfy

*Corresponding Author: Xuemei Zhang, School of Mathematics and Physics, North China Electric Power University, Beijing, 102206, PR China, E-mail: zxm74@sina.com

Meiqiang Feng, School of Applied Science, Beijing Information Science \& Technology University, Beijing, 100192, PR China, E-mail: meiqiangfeng@sina.com 
(K): $K \in C^{\infty}(\Omega)$ and $K(x)>0$ in $\Omega$;

(f1) : there exists $\eta \in \mathbb{R}^{1} \cup\{-\infty\}$ such that

(i) $f \in C^{\infty}(\eta, \infty)$ is positive and strictly increasing in $(\eta, \infty)$,

(ii) if $\eta \in \mathbb{R}^{1}$ then additionally $f(\eta):=\lim _{s \rightarrow \eta} f(s)=0$.

The boundary blow-up problems were first studied by Cheng and Yau [21, 22] with $f(u)$ an exponential function of $u$, due to their applications in geometry. The case $f(u)=u^{p}(p>0)$ and $K(x)$ is a smooth positive function over $\bar{\Omega}$ was considered by Lazer and McKenna [23]. Further results can be found in [24-32], and especially papers [33-36] which have mainly motivated us.

Mohammed [33] proved that if $K(x)$ satisfies (K) and is such that the Dirichlet problem

$$
M[u]=K(x) \text { in } \Omega, u=0 \text { on } \partial \Omega
$$

has a strictly convex solution, then (1.1) has a strictly convex solution if $f$ satisfies (f1) and the Keller-Osserman ([37] and [38]) type condition

$$
\Psi(r)=\int_{r}^{\infty}[(N+1) F(s)]^{-1 /(N+1)} d s<\infty, \forall r>\eta .
$$

Here

$$
F(s)=\int_{\eta}^{s} f(t) d t \text { if } \eta \in \mathbb{R}^{1}, F(s)=\int_{0}^{s} f(t) d t \text { if } \eta=-\infty .
$$

In [34], the authors showed that, in the case that $\eta>-\infty$, (1.3) alone does not guarantee the existence of a strictly convex solution to (1.1). One needs additionally

$$
\int_{\eta^{+}}[(N+1) F(s)]^{-1 /(N+1)} d s=\infty .
$$

Here $\int_{\eta^{+}} \Phi(s) d s=\infty$ means that

$$
\int_{\eta}^{\eta+\epsilon} \Phi(s) d s=\infty \text { for all small positive } \epsilon .
$$

Obviously, (1.4) is equivalent to $\lim _{r \rightarrow \eta^{+}} \Psi(r)=\infty$.

From Theorem 1.1 and Theorem 1.2 of [34] we know, if $K(x)$ satisfies (K) and $K \in L^{\infty}(\Omega)$, the KellerOsserman type condition is necessary and sufficient (combing with (1.4) if $\eta \in \mathbb{R}^{1}$ ) for the existence of strictly convex solution, but if $K(x)$ satisfies $(\mathbf{K})$ and is such that (1.2) has a strictly convex solution, it is only sufficient. We would like to prove the necessity in this paper.

So the first main result of this paper is the following.

Theorem 1.1. Suppose that $K(x)$ satisfies $(\mathbf{K})$ and is such that (1.2) has a strictly convex solution. Suppose that $f(u)$ satisfies (f1), and when $\eta \in \mathbb{R}^{1}$, it satisfies additionally (1.4). Then (1.1) has a strictly convex solution if and only if (1.3) holds.

At the same time, in [34], the authors did not consider the boundary asymptotic behavior of the strictly convex solution. The study of boundary asymptotic behavior of blow-up solutions is also a hot topic, see [37-51], and the references therein. Recently, in [35], Zhang studied the boundary behavior of the strictly convex solution to (1.1) with $K(x) \in C(\bar{\Omega})$. Very recently, in [36], Zhang studied the boundary behavior of the strictly convex solution to (1.1) with $f(u)$ including gradient terms and $K(x)$ is in general case or borderline case.

In [35] and [36], there is an important condition on $f$, i.e.

(f2) there exists $C_{f} \in(0, \infty]$ such that

$$
\lim _{s \rightarrow \infty} H^{\prime}(s) \int_{s}^{\infty} \frac{d \tau}{H(\tau)}=C_{f}, \quad H(s):=[(N+1) F(s)]^{1 /(N+1)}, \forall s>0 .
$$


However, we find that it is not necessary. It is implied by other conditions. By Lemma AP.2 in Appendix we can see (f1) and (1.3) imply (f2).

Let

$$
I(s)=\frac{\Psi^{\prime \prime}(s) \Psi(s)}{\left(\Psi^{\prime}(s)\right)^{2}}, \quad I_{\infty}=\lim _{s \rightarrow \infty} I(s)
$$

We can see $C_{f}$ has the same meaning of $I_{\infty}$.

By Theorem 1.1 the existence of strictly convex solution to problem (1.2) is the key point for the existence of strictly convex solution to problem (1.1). If $K(x)$ is bounded on $\Omega$, according to Theorem 1 of [52], (1.2) always has a strictly convex solution. If $K(x)$ is unbounded near $\partial \Omega$, problem (1.2) is not always having solution. The existence of solution depends on the increasing speed of $K(x)$ when $x$ approaches $\partial \Omega$. In [34], the authors gave a sufficient condition for the existence of strictly convex solutions. For ease of composition, we first introduce some notations.

For a positive function $p(t)$ in $C^{1}(0, \infty)$ satisfying $p^{\prime}(t)<0$ and $\lim _{t \rightarrow 0^{+}} p(t)=+\infty$, to distinguish its behavior near $t=0$ we set $P(\tau)=\int_{\tau}^{1} p(t) d t$. We say such a function $p(t)$ is of class $\mathcal{P}_{\text {finite }}$ if

$$
\int_{0^{+}}[P(\tau)]^{\frac{1}{N}} d \tau<\infty
$$

and is of class $\mathcal{P}_{\infty}$ if

$$
\int_{0^{+}}[P(\tau)]^{\frac{1}{N}} d \tau=\infty .
$$

In Theorem 1.5 of [34], the author proved that, if $K(x)$ satisfies (K), then (1.2) has no strictly convex solution if there exists a function $p(t)$ of class $\mathcal{P}_{\infty}$ such that $K(x) \geq p(d(x))$ near $\partial \Omega$, and has a strictly convex solution if there exists a function $p(t)$ of class $\mathcal{P}_{\text {finite }}$ such that $K(x) \leq p(d(x))$ near $\partial \Omega$.

If $p(t)$ is of class $\mathcal{P}_{\text {finite }}$, we may modify $p(t)$ for large $t$ and assume that $p(t)=c_{0} e^{-t}$ for some positive constant $c_{0}$ and all large $t$, say $t \geq M_{0}$. With $p(t)$ modified as above, if we define

$$
\tilde{P}(\tau)=\int_{\tau}^{\infty} p(t) d t
$$

then we still have

$$
\int_{0^{+}}[\tilde{P}(\tau)]^{\frac{1}{N}} d \tau<\infty
$$

Moreover,

$$
\tilde{P}(t)=c_{0} e^{-t}, \tilde{P}(t) / p(t)=1 \text { for } t \geq M_{0}, \tilde{P}(t) / p(t) \rightarrow 0 \text { as } t \rightarrow 0 \text {. }
$$

Set

$$
\omega(t):=\int_{0}^{t}(N \tilde{P}(\tau))^{\frac{1}{N}} d \tau \text { for } t>0
$$

Let

$$
J(s)=-\frac{\omega(s) \omega^{\prime \prime}(s)}{\left(\omega^{\prime}(s)\right)^{2}}, J_{0}=\lim _{s \rightarrow 0^{+}} J(s) .
$$

The second main result of this paper is the following.

Theorem 1.2. Suppose that $K(x)$ satisfies $(\mathbf{K})$ and there exists a function $p(t)$ of class $\mathcal{P}_{\text {finite }}$ such that

$$
k_{2} p(d(x)) \leq K(x) \leq k_{1} p(d(x)) \text { near } \partial \Omega,
$$

where $k_{1}, k_{2}$ are positive constants. Suppose that $f(u)$ satisfies (f1), (1.3) and when $\eta \in \mathbb{R}^{1}$, it satisfies additionally (1.4). $f, K$ are such that $I_{\infty}=\infty$ and $J_{0}=\infty$ can not hold at same time, then for any strictly convex solution $u(x)$ of (1.1), it holds

$$
1 \leq \liminf _{\substack{x \in \Omega, d(x) \rightarrow 0}} \frac{u(x)}{\psi\left[\underline{\xi}(\omega(d(x)))^{\frac{N}{N+1}}\right]}, \limsup _{\substack{x \in \Omega, d(x) \rightarrow 0}} \frac{u(x)}{\psi\left[\bar{\xi}(\omega(d(x)))^{\frac{N}{N+1}}\right]} \leq 1,
$$


where $\psi$ is the inverse of $\Psi$, i.e. $\psi$ satisfies

$$
\int_{\psi(t)}^{\infty}[(N+1) F(s)]^{-1 /(N+1)} d s=t, \forall t>\eta
$$

and

$$
\begin{aligned}
& \bar{\xi}=\left\{\frac{(N+1)^{N} k_{2}}{N^{N} M_{0}\left[\frac{N}{N+1} \frac{1}{J_{0}}+\frac{1}{(N+1) J_{0} I_{\infty}}+\frac{1}{I_{\infty}}\right]}\right\}^{\frac{1}{N+1}}, \\
& \underline{\xi}=\left\{\frac{(N+1)^{N} k_{1}}{N^{N} m_{0}\left[\frac{N}{N+1} \frac{1}{J_{0}}+\frac{1}{(N+1) J_{0} I_{\infty}}+\frac{1}{I_{\infty}}\right]}\right\}^{\frac{1}{N+1}} .
\end{aligned}
$$

Here

$$
\begin{aligned}
& M_{0}=\max _{\bar{x} \in \partial \Omega} \kappa_{1}(\bar{x}) \kappa_{2}(\bar{x}) \ldots \kappa_{N-1}(\bar{x}), \\
& m_{0}=\min _{\bar{x} \in \partial \Omega} \kappa_{1}(\bar{x}) \kappa_{2}(\bar{x}) \ldots \kappa_{N-1}(\bar{x}),
\end{aligned}
$$

$\kappa_{1}(\bar{x}), \kappa_{2}(\bar{x}), \ldots \kappa_{N-1}(\bar{x})$ are the principal curvatures of $\partial \Omega$ at $\bar{x}$.

Corollary 1.3. In Theorem 1.2, if $\Omega$ is a ball of radius $R, I_{\infty} \in[1, \infty)$ and $k_{1}=k_{2}=\bar{k}$, then

$$
\lim _{\substack{x \in \Omega, d(x) \rightarrow 0}} \frac{u(x)}{\psi\left[(\omega(d(x)))^{\frac{N}{N+1}}\right]}=\xi_{0}^{1-I_{\infty}},
$$

where

$$
\xi_{0}=\left\{\frac{\bar{k} R^{N-1}}{\left(\frac{N}{N+1}\right)^{N}\left[\frac{N}{N+1} \frac{1}{J_{0}}+\frac{1}{N+1} \frac{1}{J_{0} I_{\infty}}+\frac{1}{I_{\infty}}\right]}\right\}^{\frac{1}{N+1}} .
$$

For more articles about boundary blow-up solutions in a ball, please see [53-57].

Remark 1.4. We can determine that the condition imposed on $b(x)$ in [36] is equivalent to

$$
\int_{0^{+}}[(N+1) p(\tau)]^{\frac{1}{N+1}} d \tau<\infty .
$$

We can see that (1.6) is a weaker condition than (1.12).

For example, letting $p(s)=s^{-N-1}(-\ln s)^{-\beta}, 0<s<s_{0}<1$, then $\tilde{P}(s) \cong s^{-N}(-\ln s)^{-\beta}$ as $s \rightarrow 0^{+}$. Then we have

$$
\begin{aligned}
& \int_{0^{+}}[(N+1) p(s)]^{\frac{1}{N+1}} d s \\
& =\int_{0^{+}}(N+1)^{\frac{1}{N+1}} s^{-1}(-\ln s)^{-\frac{\beta}{N+1}} d s \\
& =\left.(N+1)^{\frac{1}{N+1}} \frac{N+1}{N+1-\beta}(-\ln s)^{\frac{N+1-\beta}{N+1}}\right|_{0^{+}} \\
& \rightarrow\left\{\begin{array}{c}
0, \beta>N+1, \quad \text { as } s \rightarrow 0^{+} . \\
\infty, \beta<N+1,
\end{array}\right. \\
& \int_{0^{+}}[N \tilde{P}(s)]^{\frac{1}{N}} d s \\
& \cong \int_{0^{+}} N^{\frac{1}{N}} s^{-1}(-\ln s)^{-\frac{\beta}{N}} d s \\
& =\left.N^{\frac{1}{N}} \frac{N}{N-\beta}(-\ln s)^{\frac{N-\beta}{N}}\right|_{0^{+}} \\
& \rightarrow\left\{\begin{array}{l}
0, \beta>N, \\
\infty, \beta<N,
\end{array} \text { as } s \rightarrow 0^{+} .\right.
\end{aligned}
$$

Meanwhile, by Theorem 1.5 of [34] we know (1.6) is sharper than (1.12) for the existence of strictly convex solutions of (1.2) and the existence of strictly convex solutions of (1.1). 
If $K(x)$ is such that (1.2) has no strictly convex solution, then (1.1) may have or have no strictly convex solution, depending on the behavior of $f$. In [34], the authors only examined some such cases for the radially symmetric situation. In this paper, we'll consider the general case. But we have to impose some sufficient condition such that (1.2) has no strictly convex solution. It is

(K1) there exists a function $p(t)$ of class $\mathcal{P}_{\infty}$ such that $k_{4} p(d(x)) \leq K(x) \leq k_{3} p(d(x))$ near $\partial \Omega$, where $k_{3}, k_{4}$ are positive constants.

Suppose that $K(x)$ satisfies $(\mathbf{K})$ and (K1), $f(u)$ satisfies (f1). We'll prove that (1.1) has strictly convex solution if (1.3) does not hold.

If (1.3) does not hold, there exists $c_{0}>0$ such that

$$
G(t):=\int_{c_{0}}^{t}[(N+1) F(\tau)]^{-\frac{1}{N+1}} d \tau \rightarrow \infty \text { as } t \rightarrow \infty .
$$

Let

$$
R(s)=-\frac{G^{\prime \prime}(s) G(s)}{\left(G^{\prime}(s)\right)^{2}}, R_{\infty}=\lim _{s \rightarrow \infty} R(s) .
$$

We have

Theorem 1.5. Suppose that $K(x)$ satisfies $(\mathbf{K})$ and (K1). Suppose that $f(u)$ satisfies $(\mathbf{f} \mathbf{1})$ and $R_{\infty} \neq \infty$. Then (1.1) has strictly convex solution if (1.3) does not hold.

The rest of the paper is organized in the following way. In Section 2 we will collect some known results to be used in the subsequent sections. Section 3 is devoted to the proofs of the Theorem 1.1 and Theorem 1.2. In Section 4 we prove that Theorem 1.5 holds. In Appendix we will introduce the theory of regular variation for the proof of Corollary 1.3.

\section{Some preliminary results}

In this section, we collect some results for the convenience of later use and reference.

Lemma 2.1. (Lemma 2.1 of [23]) Let $\Omega$ be a bounded domain in $\mathbb{R}^{N}, N \geq 2$, and let $u^{k} \in C^{2}(\Omega) \cap C(\bar{\Omega})$ for $k=1$, 2. Let $f(x, u)$ be defined for $x \in \Omega$ and $u$ in some interval containing the ranges of $u^{1}$ and $u^{2}$ and assume that $f(x, u)$ is strictly increasing in $u$ for all $x \in \Omega$. Suppose

(i) the matrix $\left(u_{x_{i} x_{j}}^{1}\right)$ is positive definite in $\Omega$,

(ii) $M\left[u^{1}\right](x) \geq f\left(x, u^{1}(x)\right), \forall x \in \Omega$,

(iii) $M\left[u^{2}\right](x) \leq f\left(x, u^{2}(x)\right), \forall x \in \Omega$,

(iv) $u^{1}(x) \leq u^{2}(x), \forall x \in \partial \Omega$.

Then $u^{1}(x) \leq u^{2}(x)$ in $\Omega$.

Remark 2.2. From the proof in [23], it is easily seen that the condition " $f(x, u)$ is strictly increasing in $u$ for all $x \in \Omega$ " in Lemma 2.1 can be relaxed to " $f(x, u)$ is nondecreasing in $u$ for all $x \in \Omega$ " provided that one of the inequalities in (ii) and (iii) is replaced by a strict inequality. This observation will be used later in the paper.

Lemma 2.3. (Proposition 2.1 of [24]) Let $u \in C^{2}(\Omega)$ be such that the matrix $\left(u_{x_{i} x_{j}}\right)$ is invertible for $x \in \Omega$, and let $g$ be a $C^{2}$ function defined on an interval containing the range of $u$. Then

$$
M[g(u)]=M[u]\left\{\left[g^{\prime}(u)\right]^{N}+\left[g^{\prime}(u)\right]^{N-1} g^{\prime \prime}(u)(\nabla u)^{T} B(u) \nabla u\right\},
$$

where $A^{T}$ denotes the transpose of the matrix $A, B(u)$ denotes the inverse of the matrix $\left(u_{x_{i} x_{j}}\right)$, and

$$
\nabla u=\left(u_{x_{1}}, u_{x_{2}}, \cdots, u_{x_{N}}\right)^{T} .
$$


If $u=d(x)$, then

$$
M[g(d(x))]=\left[-g^{\prime}(d(x))\right]^{N-1} g^{\prime \prime}(d(x)) \prod_{i=1}^{N-1} \frac{\kappa_{i}(\bar{x})}{1-d(x) \kappa_{i}(\bar{x})}, x \in \Omega_{\delta_{1}},
$$

where

$$
\Omega_{\delta_{1}}=\left\{x \in \Omega: 0<d(x)<\delta_{1}\right\}, \quad \kappa_{1}(\bar{x}), \kappa_{2}(\bar{x}), \ldots \kappa_{N-1}(\bar{x})
$$

are the principal curvatures of $\partial \Omega$ at $\bar{x}$.

The following interior estimate for derivatives of smooth solutions of Monge-Ampère equations is a simple variant of Lemma 2.2 in [23], which follows from [58, 59].

Lemma 2.4. Let $\Omega$ be a bounded domain in $\mathbb{R}^{N}, N \geq 2$, with $\partial \Omega \in C^{\infty}$. Let $\eta \in[-\infty,+\infty)$ and $f \in C^{\infty}(\bar{\Omega} \times(\eta, \infty))$ with $f(x, u)>0$ for $(x, u) \in \bar{\Omega} \times(\eta, \infty)$. Let $u \in C^{\infty}(\bar{\Omega})$ be a solution of the Dirichlet problem

$$
\begin{cases}M[u](x)=f(x, u), & x \in \Omega, \\ u(x)=c=\text { constant }, & x \in \partial \Omega,\end{cases}
$$

with $\eta<u(x)<$ c in $\Omega$. Let $\Omega^{\prime}$ be a subdomain of $\Omega$ with $\overline{\Omega^{\prime}} \subset \Omega$ and assume that $\eta<a \leq u(x) \leq b$ for $x \in \overline{\Omega^{\prime}}$ and let $k \geq 1$ be an integer. Then there exists a constant $C$ which depends only on $k, a, b, b o u n d s$ for the derivatives of $f(x, u)$ for $(x, u) \in \overline{\Omega^{\prime}} \times[a, b]$, and $\operatorname{dist}\left(\Omega^{\prime}, \partial \Omega\right)$ such that

$$
\|u\|_{C^{k}\left(\overline{\Omega^{\prime}}\right)} \leq C .
$$

The existence result below is a variant of Lemma 2.3 in [23], which is a special case of Theorem $7.1 \mathrm{in}$ [52].

Lemma 2.5. Let $\Omega$ be a strictly convex, bounded domain in $\mathbb{R}^{N}, N \geq 2$, with $\partial \Omega \in C^{\infty}$. Let $f(x, u)$ be a positive $C^{\infty}$ function on $\bar{\Omega} \times(\eta, c]$, where $c>\eta \geq-\infty$. If there exists a function $u_{\star} \in C^{2}(\bar{\Omega})$, which is convex on $\bar{\Omega}$, such that $u_{\star}>\eta$ and

$$
\begin{cases}M\left[u_{\star}\right](x) \geq f\left(x, u_{\star}(x)\right), & x \in \Omega, \\ u_{*}(x)=c, & x \in \partial \Omega,\end{cases}
$$

then there exists a solution $u$ of (2.3) with $u \in C^{\infty}(\bar{\Omega})$ and $u$ strictly convex. Moreover, $u(x) \geq u_{\star}(x)$ on $\bar{\Omega}$.

Let $\Omega$ be a smooth, bounded, strictly convex domain in $\mathbb{R}^{N}$, by Theorem 1.1 of [52], there exists $u_{0} \in C^{\infty}(\bar{\Omega})$ which is the unique strictly convex solution to

$$
M\left[u_{0}\right]=1 \text { in } \Omega, u_{0}=1 \text { on } \partial \Omega .
$$

Set $z(x):=1-u_{0}(x)$. Then $z(x)>0$ in $\Omega$ and it is the unique strictly concave solution to

$$
(-1)^{N} M[z]=1 \text { in } \Omega, z=0 \text { on } \partial \Omega \text {. }
$$

Since $\left(z_{x_{i} x_{j}}\right)$ is negative definite on $\bar{\Omega}$, its trace is negative, that is $\Delta z<0$, and hence one can apply the Hopf boundary lemma to conclude that $|\nabla z|>0$ for $x \in \partial \Omega$. It follows that there exist positive constants $b_{1}$ and $b_{2}$ such that

$$
b_{1} d(x) \leq z(x) \leq b_{2} d(x) \text { for } x \in \Omega .
$$

\section{Proof of Theorem 1.1 and Theorem 1.2}

\section{Proof of Theorem 1.1.}

Sufficiency. It was proved in [34].

Necessity. Assume to the contrary that (1.1) has a strictly convex solution $u$. We aim to derive a contradiction. 
Denote by $g(t)$ the inverse of $G(t)$, i.e.,

$$
\int_{c_{0}}^{g(t)}[(N+1) F(\tau)]^{-\frac{1}{N+1}} d \tau=t, \forall t>0,
$$

where $G(t)$ is defined by (1.13).

Then

$$
g(0)=c_{0}, \lim _{t \rightarrow \infty} g(t)=\infty
$$

and

$$
\begin{gathered}
g^{\prime}(t)=[(N+1) F(g(t))]^{\frac{1}{N+1}}, \quad g^{\prime \prime}(t)=\frac{f(g(t))}{[(N+1) F(g(t))]^{N-1}}, \\
\left(g^{\prime}(t)\right)^{N-1} g^{\prime \prime}(t)=f(g(t)), \quad \frac{g^{\prime}(t)}{g^{\prime \prime}(t)}=\frac{[(N+1) F(g(t))]^{\frac{N}{N+1}}}{f(g(t))} .
\end{gathered}
$$

Since $\Omega$ is bounded in $R^{N}$, there exists $R_{0}$ such that $\Omega \subset B\left(0, R_{0}\right)$. Then define

$$
y(x):=\sum_{i=1}^{N} \frac{1}{2}\left(x_{i}+R_{0}+K_{1}\right)^{2} \text { for } x \in \Omega,
$$

where $K_{1}$ is a positive constant to be determined.

Clearly

$$
[\nabla y(x)]^{T}=\left(x_{1}+R_{0}+K_{1}, \ldots, x_{N}+R_{0}+K_{1}\right)>0,
$$

$\left(y_{x_{i} x_{j}}\right)$ is the identity matrix, and $M[y]=1$.

Let $w_{1}(x)$ be a strictly convex solution of (1.2) and $K_{1}=\max _{x \in \bar{\Omega}}\left|\nabla w_{1}\right|+1$. Let

$$
w(x)=w_{1}(x)+y(x)+H,
$$

where $H=\max _{x \in \bar{\Omega}}\left(-w_{1}(x)\right)+1$. Then

$$
|\nabla w|=\left|\nabla w_{1}+\nabla y\right|>1, M[w]>M\left[w_{1}\right]
$$

For $c>0$ define

$$
v(x):=g(c w(x)), x \in \Omega .
$$

Then we obtain, for $x \in \Omega$,

$$
\begin{aligned}
M[v] & =M[c w]\left\{\left[g^{\prime}(c w)\right]^{N}+\left(g^{\prime}(c w)\right)^{N-1} g^{\prime \prime}(c w)(\nabla(c w))^{T} B(c w) \nabla(c w)\right\} \\
& =c^{N} M[w]\left(g^{\prime}(c w)\right)^{N-1} g^{\prime \prime}(c w)\left\{\frac{g^{\prime}(c w)}{g^{\prime \prime}(c w)}+c(\nabla w)^{T} B(w) \nabla w\right\} \\
& >c^{N} M\left[w_{1}\right] f(v)\left\{\frac{g^{\prime}(c w)}{g^{\prime \prime}(c w)}+c \lambda_{1}|\nabla w|^{2}\right\} \\
& >c^{N+1} \lambda_{1} K(x) f(v),
\end{aligned}
$$

where $B(w)$ is the inverse matrix of $\left(w_{x_{i} x_{j}}\right), \lambda_{1}$ is the minimal eigenvalue of $B(w)$. Since $w$ is strictly convex, all the eigenvalue of $B(w)$ is positive. We thus obtain

$$
M[v]>K(x) f(v) \text { in } \Omega
$$

provided that $c$ is chosen large enough.

Fix $x_{1} \in \Omega$ and by further enlarging $c$ if necessary we may assume that

$$
v\left(x_{1}\right)>u\left(x_{1}\right) \text { and } M[v]>K(x) f(v) \text { in } \Omega .
$$


Since $u(x) \rightarrow \infty$ as $d(x) \rightarrow 0$, while $v(x)$ is continuous on $\bar{\Omega}$, there exists an open connected set $D$ such that $x_{1} \in D, \bar{D} \subset \Omega, u(x)<v(x)$ in $D$ and $u(x)=v(x)$ on $\partial D$.

On the other hand, since

$$
M[u]=K(x) f(u) \text { in } D \text { and } v=u \text { on } \partial D,
$$

and the matrix $\left(v_{x_{i} x_{j}}\right)$ is positive definite on $\bar{D}$ (since $w_{1}(x), y(x)$ are strictly convex in $\Omega$ and $g^{\prime}, g^{\prime \prime}>0$ ), we can apply Lemma 3.1 to conclude that $v(x) \leq u(x)$ in $D$. This contradiction completes our proof.

\section{Proof of Theorem 1.2.}

For small $\delta_{1}>0$, let

$$
\Omega_{\delta_{1}}=\left\{x \in \Omega \mid 0<d(x)<\delta_{1}\right\} .
$$

For an arbitrary $\varepsilon \in\left(0, \min \left\{1 / 4, \underline{k_{2}}\right\}\right)$, let

$$
\begin{aligned}
& \bar{\xi}_{\varepsilon}=\left\{\frac{k_{2}(1-\varepsilon)^{2}}{\left(\frac{N}{N+1}\right)^{N} M_{0}\left[\frac{N}{N+1} \frac{1}{J_{0}}+\frac{1}{N+1} \frac{1}{J_{0} I_{\infty}}+\frac{1}{I_{\infty}}\right]}\right\}^{\frac{1}{N+1}}, \\
& \underline{\xi}_{\varepsilon}=\left\{\frac{k_{1}(1+\varepsilon)^{2}}{\left(\frac{N}{N+1}\right)^{N} m_{0}\left[\frac{N}{N+1} \frac{1}{J_{0}}+\frac{1}{N+1} \frac{1}{J_{0} I_{\infty}}+\frac{1}{I_{\infty}}\right]}\right\}^{\frac{1}{N+1}} .
\end{aligned}
$$

where $m_{0}, M_{0}, k_{1}, k_{2}$ are given in Theorem 1.2, $I_{\infty}, J_{0}$ are given (1.5) and (1.9).

From the definition of $J_{0}, I_{\infty}, \bar{\xi}_{\varepsilon}, \underline{\xi}_{\varepsilon}$ we see that

$$
\begin{gathered}
\lim _{d(x) \rightarrow 0} \frac{[N \tilde{P}(d(x))]^{\frac{N+1}{N}}}{p(d(x)) \int_{0}^{d(x)}(N \tilde{P}(\tau))^{\frac{1}{N}} d \tau}=\frac{1}{J_{0}}, \\
\lim _{s \rightarrow \infty} \frac{[(N+1) F(s)]^{\frac{N}{N+1}}}{f(s) \Psi(s)}=\frac{1}{I_{\infty}}, \\
\lim _{d(x) \rightarrow 0} \prod_{i=1}^{N-1}\left(1-d(x) \kappa_{i}(\bar{x})\right)=1, \\
\bar{\xi}_{\varepsilon}^{N+1}\left(\frac{N}{N+1}\right)^{N}\left[\frac{N}{N+1} \frac{1}{J_{0}}+\frac{1}{N+1} \frac{1}{J_{0} I_{\infty}}+\frac{1}{I_{\infty}}\right] \frac{M_{0}}{k_{2}(1-\varepsilon)}-1=-\varepsilon, \\
\underline{\xi}_{\varepsilon}^{N+1}\left(\frac{N}{N+1}\right)^{N}\left[\frac{N}{N+1} \frac{1}{J_{0}}+\frac{1}{N+1} \frac{1}{J_{0} I_{\infty}}+\frac{1}{I_{\infty}}\right] \frac{m_{0}}{k_{1}(1+\varepsilon)}-1=\varepsilon .
\end{gathered}
$$

For $x \in \Omega_{\delta_{1}}$, define

$$
\begin{aligned}
& \bar{u}_{\varepsilon}(x)=\psi\left[\bar{\xi}_{\varepsilon}[\bar{\omega}(d(x))]\right], \bar{\omega}(d(x))=[\omega(d(x))]^{\frac{N}{N+1}}-[\omega(\sigma)]^{\frac{N}{N+1}}, d(x)>\sigma>0, \\
& \underline{u}_{\varepsilon}(x)=\psi\left[\underline{\xi}_{\varepsilon}[\omega(d(x))]\right], \underline{\omega}(d(x))=[\omega(d(x))]^{\frac{N}{N+1}}+[\omega(\sigma)]^{\frac{N}{N+1}}, \sigma \in\left(0, \delta_{\varepsilon}\right),
\end{aligned}
$$

where $\delta_{\varepsilon} \in\left(0, \min \left\{1, \delta_{1} / 2\right\}\right)$ is sufficiently small such that for $x \in \Omega_{2 \delta_{\varepsilon}}$

$$
1-\varepsilon<\prod_{i=1}^{N-1}\left(1-d(x) \kappa_{i}(\bar{x})\right)<1+\varepsilon,
$$

$$
\begin{aligned}
& \bar{\xi}_{\varepsilon}^{N+1}\left(\frac{N}{N+1}\right)^{N}\left[\frac{N}{N+1} \frac{1}{J(d(x))}+\frac{1}{N+1} \frac{1}{I\left(\bar{u}_{\varepsilon}\right)} \frac{1}{J(d(x))}+\frac{1}{I\left(\bar{u}_{\varepsilon}\right)}\right] \frac{M_{0}}{k_{2}(1-\varepsilon)}-1<0, \\
& \underline{\xi}_{\varepsilon}^{N+1}\left(\frac{N}{N+1}\right)^{N}\left[\frac{N}{N+1} \frac{1}{J(d(x))}+\frac{1}{N+1} \frac{1}{I\left(\underline{u}_{\varepsilon}\right)} \frac{1}{J(d(x))}+\frac{1}{I\left(\underline{u}_{\varepsilon}\right)}\right] \frac{m_{0}}{k_{1}(1+\varepsilon)}-1>0 .
\end{aligned}
$$

Let

$$
D_{\sigma}^{-}=\Omega_{2 \delta_{\varepsilon} / \bar{\Omega}_{\sigma}}, \quad D_{\sigma}^{+}=\Omega_{2 \delta_{\varepsilon}-\sigma} .
$$


By (2.2) we have for $x \in D_{\sigma}^{-}$

$$
\begin{aligned}
& M\left[\bar{u}_{\varepsilon}\right]-K(x) f\left(\bar{u}_{\varepsilon}\right) \\
& =\left(\frac{N}{N+1}\right)^{N} \bar{\xi}_{\varepsilon}^{N+1}(-1)^{N-1} \psi^{\prime N-1} \psi^{\prime \prime} \omega^{\prime N-1} \omega^{\prime \prime}\left[\frac{N}{N+1} \frac{\omega^{\prime 2}}{\omega \omega^{\prime \prime}}-\frac{1}{N+1} \frac{\psi^{\prime}}{\psi^{\prime \prime} \bar{\xi}_{\varepsilon} \omega \frac{N}{N+1}} \frac{\omega^{\prime 2}}{\omega^{\prime \prime} w}+\frac{\psi^{\prime}}{\psi^{\prime \prime} \bar{\xi}_{\varepsilon} \omega \frac{N}{N+1}}\right] \\
& \prod_{i=1}^{N-1} \frac{\kappa_{i}(\bar{x})}{1-d(x) \kappa_{i}(\bar{x})}-K(x) f\left(\bar{u}_{\varepsilon}\right) \\
& =\left(\frac{N}{N+1}\right)^{N} \bar{\xi}_{\varepsilon}^{N+1} f(\psi) p(d(x))\left[-\frac{N}{N+1} \frac{\omega^{\prime 2}}{\omega \omega^{\prime \prime}}+\frac{1}{N+1} \frac{\psi^{\prime}}{\psi^{\prime \prime} \bar{\xi}_{\varepsilon} \omega \frac{N}{N+1}} \frac{\omega^{\prime 2}}{\omega^{\prime \prime} w}-\frac{\psi^{\prime}}{\psi^{\prime \prime} \bar{\xi}_{\varepsilon} \omega^{\frac{N}{N+1}}}\right] \\
& \prod_{i=1}^{N-1} \frac{\kappa_{i}(\bar{x})}{1-d(x) \kappa_{i}(\bar{x})}-K(x) f\left(\bar{u}_{\varepsilon}\right) \\
& <\left(\frac{N}{N+1}\right)^{N} \bar{\xi}_{\varepsilon}^{N+1} f(\psi) p(d(x))\left[\frac{N}{N+1} \frac{[N \tilde{P}(d(x))]^{\frac{N+1}{N}}}{\left.p(d(x)) \int_{0}^{d(x)}(N \tilde{P}(\tau))\right)^{\frac{1}{N}} d \tau}\right. \\
& \left.+\frac{1}{N+1} \frac{[(N+1) F(\psi)]]^{N+1}}{f(\psi) \Psi(\psi)} \frac{[N \tilde{P}(d(x))]^{\frac{N+1}{N}}}{\left.p(d(x)) \int_{0}^{d(x)}(N \tilde{P}(\tau))\right)^{\frac{1}{N}} d \tau}+\frac{[(N+1) F(\psi)] \frac{N}{N+1}}{f(\psi) \Psi(\psi)}\right] \prod_{i=1}^{N-1} \frac{\kappa_{i}(\bar{x})}{1-d(x) \kappa_{i}(\bar{x})}-K(x) f\left(\bar{u}_{\varepsilon}\right) \\
& =\left[\bar{\xi}_{\varepsilon}^{N+1}\left(\frac{N}{N+1}\right)^{N}\left[\frac{N}{N+1} \frac{1}{J(d(x))}+\frac{1}{N+1} \frac{1}{I(\bar{u})} \frac{1}{J(d(x))}+\frac{1}{I(\bar{u})}\right] \frac{M_{0}}{k_{2}(1-\varepsilon)}-1\right] K(x) f\left(\bar{u}_{\varepsilon}\right)<0,
\end{aligned}
$$

i.e. $\bar{u}_{\varepsilon}$ is a supersolution to (1.1) in $D_{\sigma}^{-}$.

Similarly, we can prove $\underline{u}_{\varepsilon}$ is a subsolution to (1.1) in $D_{\sigma}^{+}$.

By (1.10) and Theorem F, (1.2) has a strictly convex solution. It follows from Theorem 1.1 that (1.1) has a strictly convex solution $u$. Let $A$ be large enough such that

$$
u \leq \bar{u}_{\varepsilon}+A \text { on } d(x)=2 \delta_{\varepsilon}
$$

and

$$
\underline{u}_{\varepsilon} \leq u+A \text { on } d(x)=2 \delta_{\varepsilon}-\sigma .
$$

By the definition of $\bar{u}_{\varepsilon}$ and $\underline{u}_{\varepsilon}$, we know that $\bar{u}_{\varepsilon}(x) \rightarrow \infty$ as $d(x) \rightarrow \sigma$ and $\left.\underline{u}_{\varepsilon}\right|_{\partial \Omega}<\left.u\right|_{\partial \Omega}$. By Lemma 2.1 we have

$$
u \leq \bar{u}_{\varepsilon}+A \text { in } D_{\sigma}^{-}
$$

and

$$
\underline{u}_{\varepsilon} \leq u+A \text { in } D_{\sigma}^{+}
$$

Then

and

$$
\frac{u(x)}{\psi\left[\bar{\xi}_{\varepsilon}[\bar{\omega}(d(x))]\right]} \leq 1+\frac{A}{\psi\left[\bar{\xi}_{\varepsilon}[\bar{\omega}(d(x))]\right]}, x \in D_{\sigma}^{-},
$$

$$
1-\frac{A}{\psi\left[\underline{\xi}_{\varepsilon}[\underline{\omega}(d(x))]\right]} \leq \frac{u(x)}{\psi\left[\underline{\xi}_{\varepsilon}[\underline{\omega}(d(x))]\right]}, x \in D_{\sigma}^{+} .
$$

Then, for $x \in D_{\sigma}^{-} \cap D_{\sigma}^{+}$, the two formulas above hold. Letting $\sigma \rightarrow 0$, then we obtain

$$
\frac{u(x)}{\psi\left[\bar{\xi}_{\varepsilon}[\omega(d(x))]^{\frac{N}{N+1}}\right]} \leq 1+\frac{A}{\psi\left[\bar{\xi}_{\varepsilon}[\omega(d(x))]^{\frac{N}{N+1}}\right]},
$$

and

$$
1-\frac{A}{\psi\left[\underline{\xi}_{\varepsilon}[\omega(d(x))]^{\frac{N}{N+1}}\right]} \leq \frac{u(x)}{\psi\left[\underline{\xi}_{\varepsilon}[\omega(d(x))]^{\frac{N}{N+1}}\right]} .
$$

Letting $d(x) \rightarrow 0, \varepsilon \rightarrow 0$, we get

$$
\limsup _{\substack{x \in \Omega, d(x) \rightarrow 0}} \frac{u(x)}{\psi\left[\bar{\xi}(\omega(d(x))) \frac{N}{N+1}\right]} \leq 1,
$$

and

$$
1 \leq \liminf _{\substack{x \in \Omega, d(x) \rightarrow 0}} \frac{u(x)}{\psi\left[\underline{\xi}(\omega(d(x))) \frac{N}{N+1}\right]} .
$$

\section{Proof of Corollary 1.3.}


By (1.11) we have

$$
\psi^{\prime}(t)=-[(N+1) F(\psi(t))]^{\frac{1}{N+1}}
$$

Then

$$
\begin{aligned}
& \lim _{t \rightarrow 0} \frac{t \psi^{\prime}(t)}{\psi(t)}=-\lim _{t \rightarrow 0} \frac{t[(N+1) F(\psi(t))] \frac{1}{N+1}}{\psi(t)} \\
& =\lim _{s \rightarrow \infty} \frac{\frac{\Psi(s)}{\Psi^{\prime}(s)}}{s}=1-\lim _{s \rightarrow \infty} \frac{\Psi(s) \Psi^{\prime \prime}(s)}{\Psi^{\prime 2}(s)}=1-I_{\infty} .
\end{aligned}
$$

It follows from Proposition AP.3 of Appendix that $\psi \in N R V_{1-I_{\infty}}$.

Combing this with Theorem 1.2 we obtain

$$
\begin{aligned}
& \lim _{\substack{x \in \Omega, d(x) \rightarrow 0}} \frac{u(x)}{\psi\left[(\omega(d(x)))^{\frac{N}{N+1}}\right]} \\
& =\lim _{\substack{x \in \Omega, d(x) \rightarrow 0}} \frac{u(x)}{\psi\left[\xi_{0}(\omega(d(x)))^{\frac{N}{N+1}}\right]} \frac{\psi\left[\xi_{0}(\omega(d(x)))^{\frac{N}{N+1}}\right]}{\psi\left[(\omega(d(x)))^{\frac{N}{N+1}}\right]} \\
& =\xi_{0}^{1-I_{\infty}} .
\end{aligned}
$$

\section{Proof of Theorem 1.5}

For the proof of Theorem 1.5, we first introduce a lemma which is about radial solutions. Let $K(x)=$ $K_{1}(r), u(x)=v(r), r=|x|, B$ is a ball with radius $R$ in $\mathbb{R}^{N}(N \geq 2)$, then

$$
\begin{cases}M[u]=K(x) f(u), & x \in B, \\ u=\infty, & x \in \partial B,\end{cases}
$$

is equivalent to

$$
\left\{\begin{array}{l}
\left(v^{\prime}\right)^{N-1} v^{\prime \prime}=r^{N-1} K_{1}(r) f(v), r \in(0, R), \\
v^{\prime}(0)=0, v(R)=\infty .
\end{array}\right.
$$

In the radially symmetric setting, the smoothness requirements for $K$ and $f$ can be greatly relaxed. But for convenience, we still use (K), (K1) and (f1). In the case (K1) can be state as:

there exist constants $d_{3}, d_{4}>0$ and a function $p(t)$ of class $\mathcal{P}_{\infty}$ such that

$$
d_{4} p(R-r) \leq K_{1}(r) \leq d_{3} p(R-r) \text { for all } r<R \text { close to R. }
$$

We modify $p(t)$ as in Section 1 and define $\sigma(t)$ by

$$
\sigma(s)=\int_{s}^{\infty}(N \tilde{P}(\tau)]^{\frac{1}{N}} d \tau,
$$

we have

$$
\sigma^{\prime}(s)=-[N \tilde{P}(s)]^{\frac{1}{N}}, \sigma^{\prime \prime}(s)=[N \tilde{P}(s)]^{\frac{1}{N}-1} p(s) .
$$

It follows that

$$
-\frac{1}{\sigma^{\prime}(s)}=[N \tilde{P}(s)]^{-\frac{1}{N}}
$$

Let

$$
T(s)=\frac{\sigma(s) \sigma^{\prime \prime}(s)}{\sigma^{\prime 2}(s)}
$$

Lemma 4.1. Suppose that $K$ satisfies (K) and (K1). Suppose that $f$ satisfies (f1) and $R_{\infty} \neq \infty$. If (1.3) does not hold, then (4.2) has infinitely many strictly convex solutions. 
Proof. Let $w(r)=g\left(c \sigma^{\frac{N}{N+1}}(y(r))\right)$ for $r \in[0, R)$, where $y(r)$ satisfies

$$
\left\{\begin{array}{l}
(-1)^{N} y^{\prime N-1} y^{\prime \prime}=r^{N-1}, r \in(0, R), \\
y^{\prime}(0)=0, y(R)=0
\end{array}\right.
$$

and $g, \sigma$ is defined by (3.1),(4.3).

Similar to the proof of Theorem 5.3 of [34], we can prove the Lemma 4.1. So we omit it here.

\section{Proof of Theorem 1.5.}

\section{Step1. Let}

$$
w(x)=g\left(c \sigma^{N+1}\left(\frac{1}{b_{2}} z(x)\right)\right), x \in \Omega,
$$

where $g, \sigma, z$ is defined by (3.1),(4.3),(2.4), respectively, and $b_{2}$ is defined in (2.5). Then by (1.14), (4.4), (4.5), (3.2) we have

$$
\begin{aligned}
& M[w]=c^{N+1} b_{2}^{-N}\left(\frac{N}{N+1}\right)^{N} g^{\prime N-1} g^{\prime \prime} \sigma^{\prime N-1} \sigma^{\prime \prime} M[z]\left\{\frac{g^{\prime}}{c \sigma^{N+1} g^{\prime \prime}} \frac{\sigma^{\prime}}{\sigma^{\prime \prime}}\right. \\
& \left.-b_{2}^{-1}\left(\frac{N}{N+1} \frac{\sigma^{\prime 2}}{\sigma \sigma^{\prime \prime}}-\frac{1}{N+1} \frac{g^{\prime}}{c \sigma^{N+1} g^{\prime \prime}} \frac{\sigma^{\prime 2}}{\sigma \sigma^{\prime \prime}}+\frac{g^{\prime}}{c \sigma^{\frac{N}{N+1}} g^{\prime \prime}}\right)(\nabla z)^{T} B(z) \nabla z\right\} \\
& =c^{N+1} b_{2}^{-N}\left(\frac{N}{N+1}\right)^{N} f(w) p\left(\frac{1}{b_{2}} z\right)\left\{\frac{1}{R(w)}\left(-\frac{\sigma^{\prime}}{\sigma^{\prime \prime}}\right)\right. \\
& \left.-b_{2}^{-1}\left(\frac{N}{N+1} \frac{1}{T(z)}-\frac{1}{N+1} \frac{1}{R(w)} \frac{1}{T(z)}+\frac{1}{R(w)}\right)(\nabla z)^{T} B(z) \nabla z\right\},
\end{aligned}
$$

where $R, T$ is defined by (1.14) and (4.5) respectively.

Since

$$
\begin{aligned}
\frac{\sigma^{\prime}(t)^{2}}{\sigma(t) \sigma^{\prime \prime}(t)}= & \frac{[N \tilde{P}(t)]^{\frac{N+1}{N}}}{p(t) \int_{t}^{\infty}[N \tilde{P}(\tau)]^{1 / N} d \tau} \\
& =\frac{\int_{t}^{\infty}(N+1)[N \tilde{P}(s)]^{1 / N} p(s) d s}{\int_{t}^{\infty}\left\{-p^{\prime}(s) \int_{s}^{\infty}[N \tilde{P}(\tau)]^{1 / N} d \tau+p(s)[N \tilde{P}(s)]^{1 / N}\right\} d s} \\
& \leq(N+1) .
\end{aligned}
$$

We have

$$
\frac{1}{R(w)}-\frac{1}{N+1} \frac{1}{R(w)} \frac{1}{T(z)} \geq 0 .
$$

Then

$$
\frac{N}{N+1} \frac{1}{T(z)}-\frac{1}{N+1} \frac{1}{R(w)} \frac{1}{T(z)}+\frac{1}{R(w)}>0 .
$$

Let

$$
\triangle_{1}=\frac{1}{R(w)}\left(-\frac{\sigma^{\prime}}{\sigma^{\prime \prime}}\right)-b_{2}^{-1}\left(\frac{N}{N+1} \frac{1}{T(z)}-\frac{1}{N+1} \frac{1}{R(w)} \frac{1}{T(z)}+\frac{1}{R(w)}\right)(\nabla z)^{T} B(z) \nabla z .
$$

By the definition of $z$ we have $\left(z_{x_{i} x_{j}}\right)$ is negative definite. It follows that there exist $e_{1}, e_{2}>0$ such that

$$
-e_{1}\|\nabla z\|^{2} \leq(\nabla z)^{T} B(z) \nabla z \leq-e_{2}\|\nabla z\|^{2},
$$

and trace $\left(z_{x_{i} x_{j}}\right)=\Delta z<0$. Therefore, since $\Delta(-z)>0$ on $\Omega$ and $-z$ attains its maximum on $\bar{\Omega}$ at each point of $\partial \Omega$, it follows from the maximum principle that there exists an open set $\mathrm{U}$ containing $\partial \Omega$ such that

$$
\|\nabla z\| \geq e>0 \text {. }
$$

On the other hand, it is easy to see that $z$ is bounded below by a positive constant on $\Omega-\mathrm{U}$. Then

$$
w \rightarrow \infty \text { as } c \rightarrow \infty \text { on } \Omega-\mathrm{U} .
$$


Combining this with the fact that $R_{\infty} \neq \infty$, we can conclude that $\Delta_{1}$ is positive on $\Omega$. By (4.6) we have

$$
\begin{aligned}
& M[w] \geq c^{N+1} b_{2}^{-N}\left(\frac{N}{N+1}\right)^{N} f(w) p(d(x)) \Delta_{1} \\
& \geq c^{N+1} b_{2}^{-N}\left(\frac{N}{N+1}\right)^{N} f(w) \frac{1}{k_{3}} K(x) \Delta_{1} \\
& \geq K(x) f(w)
\end{aligned}
$$

for large $c_{1}$, i.e. $w_{1}(x)=g\left(c_{1} \sigma^{\frac{N}{N+1}}\left(\frac{1}{b_{2}} z(x)\right)\right)$ is a subsolution of (1.1).

Step2. The existence of a solution $u(x) \in C^{\infty}(\Omega)$.

Let $\left\{\sigma_{n}\right\}_{1}^{\infty}$ be a strictly increasing sequence of positive numbers such that $\sigma_{n} \rightarrow \infty$ as $n \rightarrow \infty$, and let $\Omega_{n}=$ $\left\{x \in \Omega \mid w_{1}(x)<\sigma_{n}\right\}$. Since any level surface of $w_{1}$ is a level surface of $z$, for each $n \geq 1, \partial \Omega_{n}$ is a strictly convex $C^{\infty}$-submanifold of $R^{N}$ of dimension $N-1$.

By Lemma 2.5 there exists $u_{n} \in C^{\infty}\left(\bar{\Omega}_{n}\right)$ for $n \geq 1$ such that

$$
\left\{\begin{array}{c}
M\left[u_{n}\right]=K(x) f\left(u_{n}\right), x \in \Omega_{n}, \\
u_{n}\left\|_{\partial \Omega_{n}}=\sigma_{n}=w_{1}\right\|_{\partial \Omega_{n}} .
\end{array}\right.
$$

By Lemma 2.1 and (4.7)

$$
u_{n}(x) \geq w_{1}(x) \quad x \in \bar{\Omega}_{n} .
$$

Clearly, for $n>1$

$$
\bar{\Omega}_{n} \subset \Omega_{n+1}
$$

and

$$
\Omega=\bigcup_{n=1}^{\infty} \Omega_{n}
$$

We claim that

$$
u_{n}(x) \leq u_{n+1}(x), \quad \forall x \in \Omega_{n} .
$$

Indeed, since $u_{n}$ and $u_{n+1}$ are both positive solutions of $M[u]=K(x) f(u)$ on $\bar{\Omega}_{n}, u_{n}$ is strictly convex in $\Omega_{n}$ and for $x \in \partial \Omega_{n} \subset \Omega_{n+1}$,

$$
u_{n+1}(x) \geq w_{1}(x)=u_{n}(x),
$$

the inequality (4.9) is a consequence of Lemma 2.1.

Fix $m$. For each $x_{0} \in \bar{\Omega}_{m}$, let $R$ be small to have $\bar{B}\left(x_{0} ; R\right) \subset \Omega_{m+1}$. By Lemma 4.1, there exists a solution $v$ of (4.2). Let $d(x, \partial B)=d\left(x, \partial B\left(x_{0} ; R\right)\right)$. Define

$$
w_{2}(x)=v(R-d(x, \partial B)), x \in B\left(x_{0} ; R\right) .
$$

Then by (2.2) and (4.2) we have

$$
\begin{aligned}
& M\left[w_{2}\right]=\left(v^{\prime}\right)^{N-1} v^{\prime \prime} \prod_{i=1}^{N-1} \frac{k_{i}(\bar{x})}{1-d(x) \kappa_{i}(\bar{x})} \\
& =r^{N-1} K_{1}(r) f\left(w_{2}\right) \prod_{i=1}^{N-1} \frac{k_{i}(\bar{x})}{1-d(x) \kappa_{i}(\bar{x})} \\
& \leq K(x) f\left(w_{2}\right)
\end{aligned}
$$

for small $R$.

It follows from Lemma $2.1 u_{n}(x) \leq w_{2}(x), x \in B\left(x_{0} ; R\right)$ for all $n \geq m+1$. Then $u_{n}$ have an uniform bound from above in $B\left(x_{0} ; R / 2\right)$ for $n \geq m+1$. Covering $\Omega_{m}$ with finite ball of this kind, one gets the uniform bound $C_{m}$, i.e. there exists $C_{m}>0$ such that

$$
u_{n}(x) \leq C_{m} \text { for } x \in \bar{\Omega}_{m}, n \geq m+1 .
$$

This implies that, for every $x \in \Omega$,

$$
u(x):=\lim _{n \rightarrow \infty} u_{n}(x) \text { exists }
$$

and

$$
u_{m}(x) \leq u(x) \leq C_{m} \text { for } x \in \bar{\Omega}_{m} .
$$


As we also have $u_{n}(x) \geq c_{0}>0$ in $\bar{\Omega}_{m}$ for $n \geq m+1$, and for such $n, \bar{\Omega}_{m} \subset \Omega_{n}$,

$$
0<\operatorname{dist}\left(\bar{\Omega}_{m}, \partial \Omega_{m+1}\right) \leq \operatorname{dist}\left(\bar{\Omega}_{m}, \partial \Omega_{n}\right)<\operatorname{dist}\left(\Omega_{m}, \partial \Omega\right),
$$

we are in a position to apply Lemma 2.4 to conclude that, for any fixed integer $k \geq 1$, there exists a constant $C=C_{k, m}$ independent of $n$ such that for all $n>m$,

$$
\left\|u_{n}\right\|_{C^{k}\left(\bar{\Omega}_{m}\right)} \leq C .
$$

It follows that the convergence $u_{n}(x) \rightarrow u(x)$ holds in $C_{l o c}^{k}(\Omega)$ for every $k \geq 1$, and $u \in C^{\infty}(\Omega)$. Moreover, for $x \in \Omega$,

$$
M[u](x)=\lim _{n \rightarrow \infty} M\left[u_{n}\right](x)=K(x) \lim _{n \rightarrow \infty} f\left(u_{n}(x)\right)=K(x) f(u(x)) .
$$

Since each $u_{n}$ is strictly convex, $u(x)$ is strictly convex in $\Omega$. Thus $u$ is a strictly convex solution of (1.1).

\section{Appendix}

We present some basic facts of Karamata regular variation theory (refer to [60], [61]) here.

Definition AP.1. A positive measurable function $f$ defined on $[A, \infty)$, for some $A>0$, is called regularly varying at infinity with index $\rho \in R$, written $f \in R V_{\rho}$, if for all $\xi>0$,

$$
\lim _{s \rightarrow \infty} \frac{f(\xi s)}{f(s)}=\xi^{\rho} .
$$

In particular, when $\rho=0, f$ is called slowly varying at infinity.

Clearly, if $f \in R V_{\rho}$, then $L(s)=\frac{f(s)}{s^{\rho}}$ is slowly varying at infinity.

Definition AP.2. A positive measurable function $f$ defined on $[A, \infty)$, for some $A>0$, is called rapidly varying at infinity if for each $\rho>1$

$$
\lim _{s \rightarrow \infty} \frac{f(s)}{s^{\rho}}=\infty
$$

or

A positive measurable function $f$ defined on $[A, \infty)$, for some $A>0$, is called rapidly varying at infinity if for each $\xi>1$

$$
\lim _{s \rightarrow \infty} \frac{f(\xi s)}{f(s)}=\infty .
$$

Proposition AP.1. (Uniform convergence theorem). If $f \in R V_{\rho}$, then (AP1) holds uniformly for $\xi \in\left[c_{1}, c_{2}\right]$ with $0<c_{1}<c_{2}$. Moreover, if $\rho<0$, then uniform convergence holds on intervals $\left(c_{1}, \infty\right)$ with $c_{1}>0$; if $\rho>0$, then uniform convergence holds on intervals $\left(0, c_{2}\right]$ provided $f$ is bounded on $\left(0, c_{2}\right]$ with $c_{2}>0$.

Proposition AP.2. (Representation theorem). A function $L$ is slowly varying at infinity if and only if it may be written in the form

$$
L(s)=\psi(s) \exp \left(\int_{A_{1}}^{s} \frac{y(\tau)}{\tau} d \tau\right), s \geq A_{1},
$$

for some $A_{1} \geq A$, where the function $\psi$ and $y$ are continuous and for $s \rightarrow \infty, y(s) \rightarrow 0$ and $\psi(s) \rightarrow c_{0}$, with $c_{0}>0$.

We say that

$$
\hat{L}(s)=c_{0} \exp \left(\int_{A_{1}}^{s} \frac{y(\tau)}{\tau} d \tau\right)
$$


is normalized slowly varying at infinity and

$$
f(s)=s^{\rho} \hat{L}(s), s \geq A_{1},
$$

is normalized regularly varying at infinity with index $\rho$ and write $f \in N R V_{\rho}$.

Proposition AP.3. A function $f \in R V_{\rho}$ belongs to $N R V_{\rho}$ if and only if

$$
f \in C^{1}\left[A_{1}, \infty\right) \text {, for some } A_{1}>0 \text { and } \lim _{s \rightarrow \infty} \frac{s f^{\prime}(s)}{f(s)}=\rho .
$$

Proposition AP.4. If function $f, g, L$ are slowly varying at infinity, then

(1) $f^{p}$ for every $p \in R, c_{1} f+c_{2} g\left(c_{1}, c_{2} \geq 0\right), f \circ g\left(\right.$ if $g(s) \rightarrow 0$ as $\left.s \rightarrow 0^{+}\right)$are also slowly varying at infinity.

(2) For every $\rho>0$ and $s \rightarrow \infty$,

$$
s^{-\rho} L(s) \rightarrow 0, s^{\rho} L(s) \rightarrow \infty .
$$

(3) For $\rho \in R$ and $s \rightarrow \infty, \frac{\ln (L(s))}{\ln s} \rightarrow 0$ and $\frac{\ln \left(s^{\rho} L(s)\right)}{\ln s} \rightarrow \rho$.

Proposition AP.5. If $f_{1} \in R V_{\rho_{1}}, f_{2} \in R V_{\rho_{2}}$, then $f_{1} f_{2} \in R V_{\rho_{1}+\rho_{2}}$ and $f_{1} \circ f_{2} \in R V_{\rho_{1} \rho_{2}}$.

Proposition AP.6. (Asymptotic behavior) If a function $L$ is slowly varying at infinity, then for $a \geq 0$ and $t \rightarrow \infty$, (1) $\int_{a}^{t} s^{\rho} L(s) d s \cong(1+\rho)^{-1} t^{1+\rho} L(t)$, for $\rho>-1$;

(2) $\int_{t}^{\infty} s^{\rho} L(s) d s \cong(-1-\rho)^{-1} t^{1+\rho} L(t)$, for $\rho<-1$.

Remark AP.1. The result of Proposition AP.6 remains true for $\rho=-1$ in the sense that

$$
\frac{\int_{a}^{t} s^{-1} L(s) d s}{L(t)} \rightarrow \infty \text { as } t \rightarrow \infty .
$$

The way to remember Proposition AP.6 is that $L(s)$ can be taken out of the integral as if it were $L(t)$, thus

$$
\int_{a}^{t} s^{\rho} L(s) d s \sim L(t) \int_{a}^{t} s^{\rho} d s(t \rightarrow \infty) .
$$

When $\rho=-1$, let $z(s)=s^{-1} L(s)$, we have

Proposition AP.7. (Asymptotic behavior)(See Karamata's Theorem 1.5.9b in [60].) If a function $z \in R V_{-1}$ and $\int_{s}^{\infty} z(\tau) d \tau<\infty, s>0$, then $\int_{s}^{\infty} z(\tau) d \tau$ is slowly varying at infinity and

$$
\lim _{s \rightarrow \infty} \frac{s z(s)}{\int_{s}^{\infty} z(\tau) d \tau}=0
$$

By (1.3) we have

$$
\Psi^{\prime}(s)=-[(N+1) F(s)]^{-\frac{1}{N+1}}, \Psi^{\prime \prime}(s)=[(N+1) F(s)]^{-\frac{1}{N+1}-1} f(s) .
$$

It follows that

$$
-\frac{1}{\Psi^{\prime}(s)}=[(N+1) F(s)]^{\frac{1}{N+1}} .
$$

By (1.5) we have

$$
I(s)=\frac{f(s) \Psi(s)}{[(N+1) F(s)]^{\frac{N}{N+1}}} .
$$

Then we have

Lemma AP.1. Let $f$ satisfy (f1) and (1.3). Then $I_{\infty} \geq 1$.

Proof. It is easy to prove by integrating $I(s)$ from $a(a>0)$ to $v$. So we omit it.

Lemma AP.2. Let $f$ satisfy (f1), (1.3). We have

(1) $I_{\infty} \in(1, \infty)$ if and only if $F \in N R V_{q+1}$ with $q>N$. In this case, $f \in R V_{q}$;

(2) $I_{\infty}=1$ if and only if $F$ is rapidly varying at infinity;

(3) $I_{\infty}=\infty$ if and only if $F \in N R V_{N+1}$. In this case, $f \in R V_{N}$.

Proof. It can be proved by the definition of regularly varying and rapidly varying at infinity. So we omit it.

Acknowledgements: This work is sponsored by the National Natural Science Foundation of China (11301178), the Beijing Natural Science Foundation (1163007), the key research and cultivation project of the improvement of scientific research level of BISTU (2018ZDPY18/521823903), and the teaching reform project of BISTU (2018JGYB32). The authors are grateful to anonymous referees for their constructive comments and suggestions, which has greatly improved this paper. 


\section{References}

[1] N.S. Trudinger and X. Wang, The Monge-Ampère equation and its geometric applications, In Handbook of geometric analysis, Vol. 1, 2008, pp. 467-524.

[2] N.S. Trudinger and X. Wang, Boundary regularity for the Monge-Ampère and affine maximal surface equations, Ann. Math. 167, (2008), 993-1028.

[3] L. Caffarelli, J.J. KOHN, L. Nirenberg, J. Spruck, The Dirichlet problem for nonlinear second-order elliptic equations II. Complex Monge -Ampère equations, and uniformly elliptic equations, Comm. Pure Appl. Math. 38, (1985), 209-252.

[4] X. Wang, Existence of Multiple Solutions to the Equations of Monge-Ampère Type, J. Differ. Equations, 100, (1992), 95-118.

[5] D. Gilbarg and N.S. Trudinger, Elliptic Partial Differential Equations of Second Order. Springer-Verlag, Berlin, Heidelberg, 2001.

[6] S. Pliś, On boundary blow-up problems for the complex Monge-Ampère equation, Proc. Amer. Math. Soc. 136, (2008), 4355-4364.

[7] Z. Zhang and K. Wang, Existence and non-existence of solutions for a class of Monge-Ampère equations, J. Differ. Equations, 246, (2009), 2849-2875.

[8] G. De Philippis and A. Figalli, Second order stability for the Monge-Ampère equation and strong Sobolev convergence of optimal transport maps, Anal. PDE. 6, (2013), 993-1000.

[9] Z. Zhang, Boundary behavior of large solutions for semilinear elliptic equations with weights, Asymptot. Anal. 96, (2016), 309-329.

[10] G. Dai, Two Whyburn type topological theorems and its applications to Monge-Ampère equations, Calc. Var. Partial Differential Equations, 55, (2016), 1-28.

[11] H. Jian, X. Wang, Y. Zhao, Global smoothness for a singular Monge-Ampère equation, J. Differ. Equations, 263, (2017), 7250-7262.

[12] Y. Li and S. Lu, Existence and nonexistence to exterior Dirichlet problem for Monge-Ampère equation, Calc. Var. Partial Differential Equations, (2018) 57: 161 https://doi.org/10.1007/s00526-018-1428-5.

[13] L. Caffarelli, Interior $W^{2, p}$ estimates for solutions of the Monge-Ampère equation, Ann. Math. 131, (1990), 135-150.

[14] S.Y. Cheng, S.T. Yau, On the regularity of the Monge-Ampère equation $\operatorname{det}\left(\left(\partial^{2} u / \partial x_{i} \partial x_{j}\right)\right)=F(x, u)$, Comm. Pure Appl. Math. 30, (1977), 41-68.

[15] M. Esteban and P. Felmer, A. Quaas, Super-linear elliptic equation for fully nonlinear operators without growth restrictions for the data, Proc. R. Soc. Edinb. 53, (2010), 125-141.

[16] G. Galise and A. Vitolo, Viscosity solutions of uniformly elliptic equations without boundary and growth conditions at infinity, Int. J. Differ. Equ. 2011, Art. ID 453727, 18 pp.

[17] A. Vitolo, M. E. Amendola, G. Galise, On the uniqueness of blow-up solutions of fully nonlinear elliptic equations, Discrete Cont. Dyn. S. 2013, (2013), 771-780.

[18] A. Mohammed, V. Rădulescu, A. Vitolo, Blow-up solutions for fully nonlinear equations: Existence, asymptotic estimates and uniqueness, Advances in Nonlinear Analysis, Article in press (2018). DOI: https://doi.org/10.1515/anona-2018-0134.

[19] I.C. Dolcetta and A. Vitolo, Glaeser's type gradient estimates for non-negative solutions of fully nonlinear elliptic equations, Discrete Cont. Dyn. S. 28, (2010), 539-557.

[20] G. Galise, S. Koike, O. Ley, A. Vitolo, Entire solutions of fully nonlinear elliptic equations with a superlinear gradient term, J. Math. Anal. Appl. 441, (2016), 194-210.

[21] S.Y. Cheng and S.T. Yau, On the existence of a complete Kähler metric on noncompact complex manifolds and the regularity of Fefferman's equation, Comm. Pure Appl. Math. 33, (1980), 507-544.

[22] S.Y. Cheng and S.T. Yau, The real Monge-Ampère equation and affine flat structures, In: Chern, S.S., Wu, W. (eds.) Proceedings of 1980 Beijing Symposium on Differential Geometry and Differential Equations, vol. 1, pp. 339-370, Beijing. Science Press, New York (1982).

[23] A.C. Lazer and P.J. McKenna, On singular boundary value problems for the Monge-Ampère operator, J. Math. Anal. Appl. 197, (1996), 341-362.

[24] F. - C. Cîrstea and C. Trombetti, On the Monge-Ampère equation with boundary blow-up: existence, uniqueness and asymptotics, Calc. Var. Partial Differential Equations, 31, (2008), 167-186.

[25] B. Guan and H. Jian, The Monge-Ampère equation with infinite boundary value, Pacific J. Math. 216, (2004), 77-94.

[26] J. Matero, The Bieberbach-Rademacher problem for the Monge-Ampère operator, Manuscripta Math. 91, (1996), $379-391$.

[27] A. Mohammed, Existence and estimates of solutions to a singular Dirichlet problem for the Monge-Ampère equation, J. Math. Anal. Appl. 340, (2008), 1226-1234.

[28] O. Savin, Pointwise $C^{2, \alpha}$ estimates at the boundary for the Monge-Ampère equation, J. Amer. Math. Soc. 26, (2013), 63-99.

[29] H. Yang, Y. Chang, On the blow-up boundary solutions of the Monge-Ampère equation with singular weights, Commun. Pure Appl. Anal. 11, (2012), 697-708.

[30] F. Cîrstea and V. Rădulescu, Blow-up boundary solutions of semilinear elliptic problems, Nonlinear Anal. 48, (2002), 521534. 
[31] F. Cîrstea and V. Rădulescu, Existence and uniqueness of blow-up solutions for a class of logistic equations, Commun. Contemp. Math. 4, (2002), no. 3, 559-586.

[32] D. Repovš, Asymptotics for singular solutions of quasilinear elliptic equations with an absorption term, J. Math. Anal. Appl. 395, (2012), 78-85.

[33] A. Mohammed, On the existence of solutions to the Monge-Ampère equation with infinite boundary values, Proc. Amer. Math. Soc. 135, (2007), 141-149.

[34] X. Zhang, Y. Du, Sharp conditions for the existence of boundary blow-up solutions to the Monge-Ampère equation, Calc. Var. Partial Differential Equations, (2018) 57:30 https://doi.org/ 10.1007/s00526-018-1312-3.

[35] Z. Zhang, Boundary behavior of large solutions to the Monge-Ampère equations with weights, J. Differ. Equations, 259, (2015), 2080-2100.

[36] Z. Zhang, Large solutions to the Monge-Ampère equations with nonlinear gradient terms: Existence and boundary behavior, J. Differ. Equations, 264, (2018), 263-296.

[37] J.B. Keller, On solutions of $\Delta u=f(u)$, Comm. Pure Appl. Math. 10, (1957), 503-510.

[38] R. Osserman, On the inequality $\Delta \geq f(u)$, Pacific J. Math. 7, (1957), 1641-1647.

[39] M. Chuaqui, C. Cortázar, M. Elgueta, C. Flores, J. García-Melián, R. Letelier, On an elliptic problem with boundary blow-up and a singular weight: the radial case, Proc. Roy. Soc. Edinburgh 133, (2003), 1283-1297.

[40] M.G. Crandall, P.H. Rabinowitz, L. Tartar, On a Dirichlet problem with a singular nonlinearity, Comm. Partial Differential Equations, 2, (1977), 193-222.

[41] X. Pan and X. Wang, Blow-up behavior of ground states of semilinear elliptic equations in $R^{n}$ involving critical sobolev exponents, J. Differ. equations, 99, (1992), 78-107.

[42] F. Cîrstea and Y. Du, General uniqueness results and variation speed for blow-up solutions of elliptic equations, Proc. London Math. Soc. 91, (2005), 459-482.

[43] F. Cîrstea and V. Rădulescu, Boundary blow-up in nonlinear elliptic equations of Bieberbach-Rademacher type, Trans. Amer. Math. Soc. 359, (2007), 3275-3286.

[44] F. Cîrstea and V. Rădulescu, Extremal singular solutions for degenerate logistic-type equations in anisotropic media, C. R. Acad. Sci. Paris, Ser. I339, (2004), 119-124.

[45] F. Cîrstea and V. Rădulescu, Nonlinear problems with boundary blow-up: a Karamata regular variation theory approach, Asymptotic Anal. 46, (2006), 275-298.

[46] J.L. Gómez, Optimal uniqueness theorems and exact blow-up rates of large solutions, J. Differ. Equations, 224, (2006), 385-439.

[47] S. Dumont, L. Dupaigne, O. Goubet, V. Rădulescu, Back to the Keller-Osserman condition for boundary blow-up solutions, Adv. Nonlinear Stud. 7, (2007), 271-298.

[48] S. Huang, W. Li, M. Wang, A unified asymptotic behavior of boundary blow-up solutions to elliptic equations, Differ. Integral Equ. 26, (2013), 675-692.

[49] S. Huang, Q. Tian, S. Zhang, J. Xi, Z. Fan, The exact blow-up rates of large solutions for semilinear elliptic equations, Nonlinear Anal. 73, (2010), 3489-3501.

[50] X. Zhang and M. Feng, Boundary blow-up solutions to the $k$-Hessian equation with singular weights, Nonlinear Anal. 167, (2018), 51-66.

[51] X. Zhang and M. Feng, Boundary blow-up solutions to the $k$-Hessian equation with a weakly superlinear nonlinearity, J. Math. Anal. Appl. 464, (2018), 456-472.

[52] L. Caffarelli, L. Nirenberg, J. Spruck, The Dirichlet problem for nonlinear second-order elliptic equations I. Monge-Ampère equations, Comm. Pure Appl. Math. 37, (1984), 369-402.

[53] I.C. Dolcetta, F. Leoni, A. Vitolo, On the inequality $F\left(x, D^{2} u\right) \geq f(u)+g(u)|D u|^{q}$, Math. Ann. 365, (2016), 423-448.

[54] A. Mohammed and G. Porru, Harnack inequality for non-divergence structure semilinear elliptic equations, Adv. Nonlinear Anal. 7, (2018), 259-269.

[55] A. Mohammed, G. Porru, A. Vitolo, Harnack inequality for nonlinear elliptic equations with strong absorption, J. Differ. Equations, 263, (2017), 6821-6843.

[56] I.C. Dolcetta, F. Leoni, A. Vitolo, On Some Degenerate Elliptic Equations Arising in Geometric Problems, J. Math. Sci. 233, (2018), 446-461.

[57] A. Vitolo, Existence of positive entire solutions of fully nonlinear elliptic equations, J. Elliptic Parabol. Equ. 4, (2018), 293304.

[58] A.V. Pogorelov, The Multidimensional Minkowski Problem, Wiley, New York, 1978.

[59] K. Tso, On a real Monge-Ampère functional, Invent. Math. 101, (1990), 425-448.

[60] N.H. Bingham, C.M. Goldie, J.L. Teugels, Regular Variation, Encyclopedia Math, Appl. vol. 27, Cambridge University Press, 1987.

[61] R. Seneta, Regular Varying Functions, Lecture Notes in Math. vol. 508, Springer-Verlag, 1976. 